\title{
A Case of a Lotus Root-like Appearance in the External Iliac Artery Detected by Intravascular Ultrasonography
}

\author{
Makoto Takeuchi ${ }^{1}$, Takenori Okada ${ }^{1}$, Takahiro Ohmine ${ }^{2}$, Yuki Ikegami ${ }^{1}$, Yumiko Nakamoto ${ }^{1}$, \\ Naomi Idei $^{1}$ and Norihiko Ohashi ${ }^{1}$
}

\begin{abstract}
:
A lotus root-like appearance on blood vessels is a rare abnormality. The multiple channels within arteries may represent the recanalization or neovascularization of the thrombus. This abnormality is most frequently found in coronary arteries. A 39-year-old woman had a thrombus-like structure in the external iliac artery. We subsequently performed an endovascular treatment six months later due to intermittent claudication. A lotus root-like appearance was found on intravascular ultrasound. To our knowledge, this is the first study to report a case of lotus root-like appearance in lower-extremity arteries.
\end{abstract}

Key words: lotus root-like appearance, external iliac artery, intravascular ultrasonography, case report

(Intern Med Advance Publication)

(DOI: 10.2169/internalmedicine.8027-21)

\section{Introduction}

A lotus root-like appearance is a rare abnormality in which percutaneous coronary intervention (PCI) may be difficult (1). This may be diagnosed via intravascular ultrasound (IVUS) and optical coherence tomography (OCT), where multiple lumens in the blood vessels with septa are present (2). Multiple channels within the coronary arteries are thought to reflect the recanalization or neovascularization of the thrombus (3).

We herein report a case of lotus root-like appearance in the external iliac artery during endovascular treatment (EVT).

\section{Case Report}

The patient was a 39-year-old woman with a height of $156 \mathrm{~cm}$, weight of $78 \mathrm{~kg}$, and body mass index of $32 \mathrm{~kg} / \mathrm{m}^{2}$. She had taken medication for type 2 diabetes mellitus for approximately 10 years. She visited our hospital because of persistent abdominal pain. An ovarian mass was identified through several imaging techniques (plain computed tomography $[\mathrm{CT}]$, magnetic resonance imaging, and positron emission tomography). Left salpingo-oophorectomy was performed the following month, and a diagnosis of stage IC ovarian cancer was made. Furthermore, at two months postoperatively, we performed total abdominal hysterectomy with right salpingo-oophorectomy because she wanted to undergo radical surgery. The ovarian cancer stage was revised to IIA, and stage IA uterine cancer was diagnosed. The patient was subsequently scheduled for a total of six courses of chemotherapy with paclitaxel and carboplatin.

After the second chemotherapy course, the patient developed sudden-onset abdominal pain. We could not detect any apparent cause of abdominal pain on contrast-enhanced CT. However, we found a thrombus in the left external iliac artery (Fig. 1). A thorough examination revealed elevated lupus anticoagulant levels (Table). After three months, the levels were still high, which was consistent with antiphospholipid syndrome (APS). The diagnosis of systemic lupus erythematosus, however, was ruled out.

In addition, we found that she had experienced pain in her left leg after walking 100 meters after taking her history. She also experienced severe pain in her left leg during a live music performance three summers ago; however, it had resolved within less than $24 \mathrm{~h}$. While she wanted her lowerextremity vessels treated, the patient was still on chemother-

${ }^{1}$ Department of Cardiology, Hiroshima Red Cross Hospital and Atomic-bomb Survivors Hospital, Japan and ${ }^{2}$ Department of Vascular Surgery, Hiroshima Red Cross Hospital and Atomic-bomb Survivors Hospital, Japan 

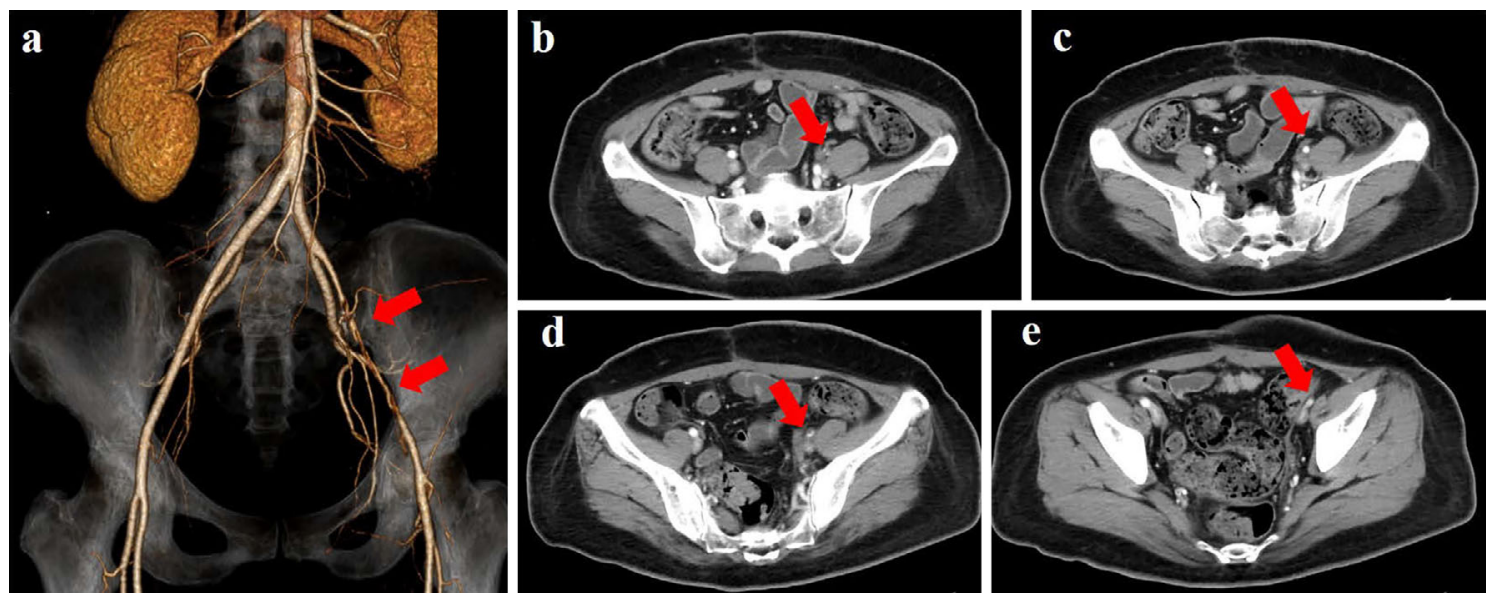

Figure 1. A thrombus-like structure in the external iliac artery on contrast-enhanced computed tomography. (a) Three-dimensional computed tomography angiography; (b) cross-section on the most head side; (c) cross-section on the most caudal side.

Table. The Patient's Laboratory Findings at the Time of Contrast Computed Tomography.

\begin{tabular}{lclc}
\hline \multicolumn{2}{l}{ Complete Blood Count } & Coagulation & \\
WBC & $3.3 \times 10^{3} / \mu \mathrm{L}$ & PT-INR & 1.16 \\
$\mathrm{RBC}$ & $4.58 \times 10^{6} / \mu \mathrm{L}$ & APTT & $34.8 \mathrm{sec}$ \\
$\mathrm{Hb}$ & $11.8 \mathrm{~g} / \mathrm{dL}$ & D-dimer & $3.0 \mu \mathrm{g} / \mathrm{mL}$ \\
$\mathrm{PLT}$ & $102 \times 10^{3} / \mu \mathrm{L}$ & LAC DRVVT & 1.39 \\
Biochemistry & & Protein C activity & 106 \\
$\mathrm{AST}$ & $19 \mathrm{IU} / \mathrm{L}$ & Protein S antigen level & 93 \\
$\mathrm{ALT}$ & $21 \mathrm{IU} / \mathrm{L}$ & Cardiolipin antibody IgG & $<8$ \\
BUN & $16.5 \mathrm{mg} / \mathrm{dL}$ & Homocysteine & $20.8 \mathrm{nmol} / \mathrm{mL}$ \\
Cre & $0.48 \mathrm{mg} / \mathrm{dL}$ & & \\
\hline
\end{tabular}

Three months later, the dilute Russell's viper venom time for the lupus anticoagulant assessment was 1.34 .

apy. As the patient wanted to prioritize chemotherapy, we continued conservative treatment with warfarin and clopidogrel for six months.

After six courses of chemotherapy, she continued to have intermittent claudication. We subsequently performed lowerextremity EVT through the right femoral artery. It was slightly difficult for us to obtain a 0.035 -inch wire through the lesion. On left lower-extremity arteriography, we found a translucent image in the left external iliac artery (Fig. 2a). We identified a lotus root-like appearance in the lesion on IVUS (Fig. 2a). We placed a $7.0 \times 120-\mathrm{mm}$ bare-metal stent (EverFlex $^{\mathrm{TM}}$ Self-expanding Peripheral Stent with Entrust ${ }^{\mathrm{TM}}$ Delivery System; Medtronic) and performed post-dilation with a $6.0 \times 80-\mathrm{mm}$ balloon. On post-treatment left lowerextremity arteriography, we found no translucent image (Fig. 2b). The ankle-brachial index improved from 0.69 to 1.10 , and the intermittent claudication disappeared.

This study was conducted in accordance with the principles embodied in the Declaration of Helsinki, and written informed consent was obtained from the patient for the publication of this case report and associated images.

\section{Discussion}

Lotus root-like appearance on the blood vessel is characterized by the presence of multiple lumens with septa, mainly diagnosed by intravascular diagnostic imaging modalities, such as IVUS and OCT (2). It has also been described as a honeycomb-like appearance. On contrast imaging, it appears braid-like $(4,5)$. Multiple channels within the coronary arteries are thought to reflect the recanalization or neovascularization of the thrombus (3). Although diabetes has been implicated in causing arteriosclerosis, our patient was too young for this complication. She did not show any atherosclerotic lesions on IVUS. In addition, she had a history of sudden left leg pain three years earlier, which led to suspicion of acute leg artery occlusion.

APS is an autoimmune disease, reported by Harris et al. in 1983 (6), which results in arterial and venous thrombosis, as well as pregnancy complications (7). The proportions of primary and secondary infections are reported to be comparable. Among patients with APS, the prevalence of thrombosis is $85.8 \%$, whereas arterial and venous thromboses are 


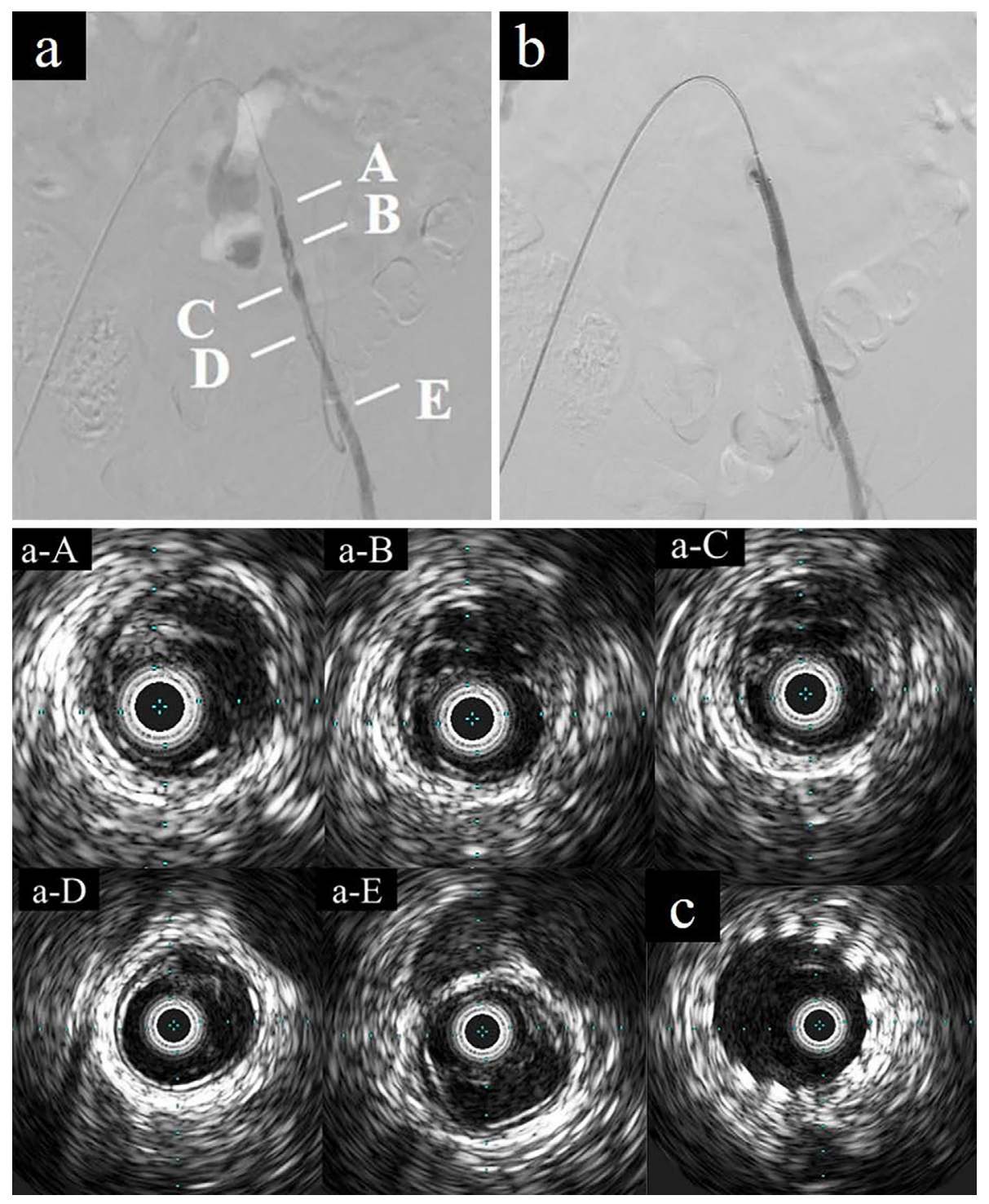

Figure 2. a: Left lower-extremity arteriography showing a translucent image in the left external iliac artery. Intravascular ultrasound showing a lotus root-like appearance. b: Post-treatment left lower-extremity arteriography showing the absence of any translucent image. c: No bare metal stent malposition was observed.

$66.0 \%$ and $32.6 \%$, respectively (8). Approximately $61 \%$ of patients with APS experience stroke, and only $2.1 \%$ have lower-extremity artery occlusion (8). Non-inferiority of direct oral anticoagulants in patients with APS has not been reported, and we chose warfarin (9). In this case, we believe that the complex interplay between APS and cancer caused such a rare condition. We cannot entirely deny the complication of fibromuscular dysplasia; nonetheless, we were unable to identify any organic abnormalities in the renal or cerebral arteries.

Most cases of lotus root-like appearance in coronary arteries have mainly been reported from Japan $(10,11)$. There exist only a few reports about lower-extremity artery. Renal and carotid arteries have already been reported $(1,12)$. However, to our knowledge, this is the first report of lotus root-like appearance in a lower-extremity artery. The renal artery report (12) ended with balloon dilation only. That pa- tient is continuing to take warfarin and clopidogrel after EVT because of APS. Since we performed total abdominal hysterectomy, we suspected that continuing warfarin would not be a major problem and decided to place a bare-metal stent. However, if similar reports in the lower-limb area are accumulated in the future, it may be possible to treat such patients with balloon dilation alone. We intend to conduct follow-up while paying attention to the symptoms of intermittent claudication and examining the ankle-brachial index.

In conclusion, with the increasing use of IVUS-guided EVT for the management of patients with chronic limbthreatening ischemia (13), the number of such reports may increase in the future.

The authors state that they have no Conflict of Interest (COI).

Acknowledgments 
The authors gratefully acknowledge the work of the past and present members of our laboratory.

\section{Funding}

The authors received no financial support for the research, authorship, and/or publication of this article.

\section{Declaration of conflicts of interests}

The authors declare that there are no conflicts of interest.

\section{References}

1. Chang H, Yoon HJ, Hong JH, Kim CH, Sohn S Il, Lee CY. A lotus root-like appearance in carotid stenosis on optical coherence tomography. BMJ Case Rep 2015: bcr2015011685, 2015.

2. Vijayvergiya R, Kasinadhuni G, Revaiah PC, Sharma P, Kumar B, Gupta A. Role of intravascular imaging for the diagnosis of recanalized coronary thrombus. Cardiovasc Revasc Med S1553-8389 (20): 30817-30814, 2020.

3. Friedman M. The coronary canalized thrombus: provenance, structure, function and relationship to death due to coronary artery disease. Br J Exp Pathol 48: 556-567, 1967.

4. Friedman M. The coronary thrombus: its origin and fate. Hum Pathol 2: 81-128, 1971.

5. Kang SJ, Nakano M, Virmani R, et al. OCT findings in patients with recanalization of organized thrombi in coronary arteries. JACC Cardiovasc Imaging 5: 725-732, 2012.

6. Harris EN, Englert H, Derve G, Hughes GR, Gharavi A. Antiphos- pholipid antibodies in acute Guillain-Barré syndrome. Lancet 2: 1361-1362, 1983.

7. Amengual O, Atsumi T. Antiphospholipid syndrome, "the best prophet of the future". Mod Rheumatol 28: 409-416, 2018.

8. Fujieda Y, Atsumi T, Amengual O, et al. Predominant prevalence of arterial thrombosis in Japanese patients with antiphospholipid syndrome. Lupus 21: 1506-1514, 2012.

9. Ordi-Ros J, Sáez-Comet L, Pérez-Conesa M, et al. Rivaroxaban Versus Vitamin K Antagonist in Antiphospholipid Syndrome: A Randomized Noninferiority Trial. Ann Intern Med 171: 658-694, 2019.

10. Terashima M, Awano K, Honda $\mathrm{Y}$, et al. "Arteries within the artery" after Kawasaki disease: a lotus root appearance by intravascular ultrasound. Circulation 106: 887, 2002.

11. Kato M, Dote K, Sasaki S. Recanalized image of thrombotic occlusion with coronary plaque rupture: a lotus root-like appearance by optical coherence tomography. Can J Cardiol 27: 871.e1-871.e 2, 2011.

12. Nakazato K, Kobayashi A, Nodera M, et al. Lotus root sign in the renal artery in renovascular hypertension. Circ J 81: 1234-1235, 2017.

13. Soga $\mathrm{Y}$, Takahara $\mathrm{M}$, Ito $\mathrm{N}$, et al. Clinical impact of intravascular ultrasound-guided balloon angioplasty in patients with chronic limb threatening ischemia for isolated infrapopliteal lesion. Catheter Cardiovasc Interv 97: E376-E384, 2021.

The Internal Medicine is an Open Access journal distributed under the Creative Commons Attribution-NonCommercial-NoDerivatives 4.0 International License. To view the details of this license, please visit (https://creativecommons.org/licenses/ by-nc-nd/4.0/).

(C) The Japanese Society of Internal Medicine Intern Med Advance Publication 